\title{
LMI-Based Intelligent Digital Redesign for Multirate Sampled-Data Fuzzy Systems
}

\author{
김도완*. 주영훈*, 박진배* \\ Do Wan Kim*, Young Hoon Joo**, and Jin Bae Park ${ }^{*}$ \\ * Yonsei University ** Kunsan University
}

\begin{abstract}
This paper presents a new linear-matrix-inequality-based intelligent digital redesign (LMI-based IDR) technique to match the states of the analog and the digital $\mathrm{T}-\mathrm{S}$ fuzzy control systems at the intersampling instants as well as the sampling ones. The main features of the proposed technique are: 1) the affine control scheme is employed to increase the degree of freedom; 2) the fuzzy-model-based periodic control is employed, and the control input is changed $n$ times during one sampling period; 3) The proposed IDR technique is based on the approximately discretized version of the $\mathrm{T}-\mathrm{S}$ fuzzy system, but its discretization error vanishes as $\mathrm{n}$ approaches the infinity. 4) some sufficient conditions involved in the state matching and the stability of the closed-loop discrete-time system can be formulated in the LMIs format.
\end{abstract}

Key words : Intelligent digital redesign (IDR), fuzzy-model-based control, digital control, fuzzy system.

\section{Introduction}

Intelligent Digital redesign (IDR) has gained tremendously increasing attention as yet another efficient de $^{-}$ sign tool of sampled-data fuzzy control [1]-[6]. The IDR problem is the problem of designing a sampled-data state feedback controller such that the sampled-data closed-loop fuzzy system is equivalent to the continuous-time closed-loop fuzzy system in the sense of the state matching.

There have been fruitful researches in the digital control system focusing on IDR method. Historically, Joo et al. first attempted to develop some intelligent digital redesign methodology for complex nonlinear systems [1]. They synergistically merged both the Takagi-Sugeno ( $\mathrm{T}$ - S) fuzzy-model-based control and the digital redesign technique for a class of nonlinear systems. Chang et al. extended the intelligent digital redesign to uncertain T-S fuzzy systems [2]. These approach [1], [2] to IDR are so called as local approach. The local approach can allows to match the states of the continuous-time and the sampled-data closed-loop fuzzy systems in the analytic way, but it may lead to undesirable and/or inaccurate results. The major reason is that the redesigned digital control gain matrices are obtained by considering only the local state-matching of each sub-closed-loop system [6]. To overcome this weakness, Lee et al. a global state-matching technique

접수일자 : 2005년 10월 21일

완료일자 : 2005년 12월 30일

This work was supported in part by the Korea Science and Engineering Foundation (Project number: R052004-000-10498-0). based on the convex optimization method, the linear matrix inequalities (LMIs) method, proposed in [6]. Specifically, their method is to globally match the states of the overall closed-loop $\mathrm{T}-\mathrm{S}$ fuzzy system with the predesigned analog fuzzy-model-based controller and those with the digitally redesigned fuzzy-model-based controller, and further to examine the stabilizability by the redesigned controller in the sense of Lyapunov. However. the IDR problem becomes the overdamped problem according as transferring the local approach to the global one in IDR problem. It may lead to undesirable and/or inaccurate results.

An affine control scheme [19] can be an alternative because the affine control scheme leads to increasing the degree of freedom. At this point, we attempt to IDR for $\mathrm{T}-\mathrm{S}$ fuzzy system based on an affine control scheme that has not yet been fully tackled under this framework. In addition, the multirate control scheme [13-18] is employed to obtain the some advantages, which allows to consider the intersampling points between sampling points and to decrease the discretization error.

Motivated by the above observations, we studies a periodic control for $\mathrm{T}-\mathrm{S}$ fuzzy systems by using the LMI-based IDR method. The main features of the proposed method are as follows: First, the affine control scheme is employed to increase the degree of freedom. Second, the fuzzy-model based periodic control is developed, and the control input is changed $n$ times during one sampling period. Second, the proposed periodic control scheme can improve the state matching performance in the long sampling limit. Finally, some sufficient conditions involved in the state matching and the stability of the closed-loop discrete-time system can be formulated in the LMIs format. 


\section{Preliminaries}

Consider a nonlinear system described by

$$
\dot{x}_{c}(t)=f\left(x_{c}(t), u_{c}(t)\right)
$$

where $x(t) \in R^{n}$ is the state vector, $u_{c}(t) \in R^{m}$ is analog control input, and the subscript " $c$ " means the analog control. To facilitate the control design, we will develop a simplified model, which can represent the local linear input-output relations of the nonlinear system. This type of models is referred as $T-S$ fuzzy models. The fuzzy dynamical model corresponding to (1) is described by the following IF - THEN rules [1]-[6]:

$$
\begin{aligned}
& R_{k}: \operatorname{IF} z_{l}(t) \text { is } \Gamma_{k l} \text { and } \cdots \text { and } z_{p}(t) \text { is } \Gamma_{k p}, \\
& \text { THEN } x_{c}(t)=A_{k} x_{c}(t)+B_{k} u_{c}(t)
\end{aligned}
$$

where $R_{k}, k \in I_{q}=\{1,2, \ldots, q\}$, is the $k$ th fuzzy rule, $z_{r}(t), r \in I_{p}=\{1,2, \ldots, p\}$, is the $r$ th premise variable, and $\Gamma_{k r},(k, r) \in I_{q} \times I_{p}$, is the fuzzy set. Then, given a pair $\left(x_{c}(t), u_{c}(t)\right)$, using the center-average defuzzification, product inference, and singleton fuzzifier, the overall dynamics of (2) has the form

$$
\dot{x}_{c}(t)=\sum_{k=1}^{q} \theta_{k}(z(t))\left(A_{k} x_{c}(t)+B_{k} u_{c}(t)\right)
$$

where $\theta_{k}(z(t))=\frac{w_{k}(z(t))}{\sum_{k=1}^{q} w_{k}(z(t))}, \quad w_{k}(z(t))=\sum_{r=1}^{p} \Gamma_{k r}\left(z_{r}(t)\right)$, and $\Gamma_{k r}\left(z_{r}(t)\right)$ is the grade of membership of $z_{r}(t)$ in $\Gamma_{k r}$. The possibly time-varying parameter vector $\theta \in R^{q}$ belongs to a convex polytope $\Theta$, where

$$
\Theta:=\left\{\sum_{k=1}^{q} \theta_{k}=1, \quad 0 \leq \theta_{k} \leq 1\right\}
$$

It is clear that as $\theta$ varies inside $\Theta, \sum_{k=1}^{q} \theta_{k}(z(t)) A_{k}$ and $\sum_{k=1}^{q} \theta_{k}(z(t)) B_{k}$ range over a matrix polytope

$$
\left[\sum_{k=1}^{q} \theta_{k}(z(t)) A_{k}, \sum_{k=1}^{q} \theta_{k}(z(t)) B_{k}\right] \in \operatorname{Co}\left\{\left(A_{k}, B_{k}\right), k \in I_{q}\right\}
$$

where Co denotes the convex hull. In this note, the stabilization of the polytopic model (3) is equivalent to the simultaneous stabilization of its vertices $\left(A_{k}, B_{k}\right), k \in I_{q}$.

In this paper, a well-constructed analog fuzzy-model-based control law, which will be employed in re- designing the digital controller. The controller is described by the following IF-THEN rules:

$$
\begin{aligned}
& R_{k}: \text { IF } z_{1}(t) \text { is } \Gamma_{k 1} \text { and } \cdots \text { and } z_{p}(t) \text { is } \Gamma_{k p}, \\
& \text { THEN } u_{c}(t)=\bar{K}_{k} x_{c}(t) \text {, }
\end{aligned}
$$

and its defuzzified output is

$$
u_{c}(t)=\sum_{k=1}^{q} \theta_{k}(z(t)) \bar{K}_{k} x_{c}(t)
$$

Therefore, main purpose of this paper is to find the digital equivalent of the following analogously controlled system:

$\dot{x}_{c}(t)=\sum_{k=1}^{q} \sum_{l=1}^{q} \theta_{k}(z(t)) \theta_{l}(z(t))\left(A_{k}+B_{k} \bar{K}_{l}\right) x_{c}(t)$

\section{Main results}

\subsection{Discretization}

In the following, let $h_{0}$ and $h$ be the sampling time and the control update time, respectively. For convenience, we take $h=\frac{h_{0}}{N}$ for a positive integer $N$, where $N$ is an input multiplicity [7]-[9]. Then, $t=i h_{0}+j h$ for $i \in Z_{0}$ and $j \in Z_{[0, N-1]}$, where the indexes $i$ and $j$ indicate sampling and control update instants, respectively.

By interfacing an ideal sampler and a zero-order holder between the plant and a controller, the digital fuzzy control system is represented by

$$
\dot{x}_{d}(t)=\sum_{k=1}^{q} \theta_{k}(z(t))\left(A_{k} x_{d}(t)+B_{k} u_{d k}(t)\right) .
$$

where $u_{d}(t)=u_{d}\left(i h_{0}+j h\right)$ for $t \in\left[i h_{0}+j h, i h_{0}+j h+h\right)$, $i \in Z_{0}, j \in Z_{[0, N-1]}$ is the periodic control input vector, the control input is changed $N$ times during one sampling time $h_{0}$, and the subscript "d" means the analog control. The periodic control input takes the following form:

$$
u_{d k}\left(i h_{0}+j h\right)=\sum_{l=1}^{q} \theta_{l}\left(z\left(i h_{0}+j h\right)\right) K_{k l} x_{d}\left(i h_{0}+j h\right)
$$

where $x_{d}\left(i h_{0}+j h\right)$ is not required to obtain $u_{d}\left(i h_{0}+j h\right)$ because it will be predicted from $x_{d}\left(i h_{0}\right)$ after each control update.

Lemma 1.[5] If $\theta_{k}(z(t))=\theta_{k}\left(z\left(i h_{0}+j h\right)\right)$ for $t \in\left[i h_{0}+j h, i h_{0}+j h+h\right), \quad i \in Z_{0}, \quad j \in Z_{[0, N-1]}$, and $\mathrm{e}^{\sum_{k=1}^{q} \theta_{k}\left(z\left(i h_{0}+j h\right)\right) A_{k} h}=\sum_{k=1}^{q} \theta_{k}\left(z\left(i h_{0}+j h\right)\right) \mathrm{e}^{A_{k} h}$ then the 
discretized system of (7) with sampling time $h$ is as follows:

$$
\begin{aligned}
& x_{d}\left(i h_{0}+j h+h\right)=\sum_{k=1}^{q} \theta_{k}\left(z\left(i h_{0}+j h\right)\right) \\
& \quad \times\left(G_{k} x_{d}\left(i h_{0}+j h\right)+H_{k} u_{d k}\left(i h_{0}+j h\right)\right)
\end{aligned}
$$

where $G_{k}=\mathrm{e}^{A_{k} h}$ and $H_{k l}=\left(G_{k}-I\right) A_{k}^{-1} B_{l}$.

In order to predict $x_{d}\left(i h_{0}+j h\right)$ in (8), we will develop a general form of solutions to (9) controlled by (8) for $x_{d}\left(i h_{0}+j h\right)$ with the arbitrary initial state $x_{d}\left(i h_{0}\right)$.

Corollary 1 . The solution to (9) closed by (8) for $x_{d}\left(i h_{0}+j h\right)$ with the arbitrary initial state $x_{d}\left(i h_{0}\right)$ is given by

$$
\begin{aligned}
& x_{d}\left(i h_{0}+j h\right)=\prod_{v=1}^{j}\left(\sum_{k=1}^{q} \sum_{l=1}^{q} \theta_{k}\left(z\left(i h_{0}+j h-v h\right)\right)\right. \\
& \left.\times \theta_{l}\left(z\left(i h_{0}+j h-v h\right)\right)\left(G_{k}+H_{k} K_{k l}\right)\right) x_{d}\left(i h_{0}\right)
\end{aligned}
$$

for $i \in Z_{0}$ and $j \in Z_{[1, N-1]}$.

Proof. The closed-loop system (9) with (8) is described by

$$
\begin{aligned}
& x_{d}\left(i h_{0}+j h+h\right)=\sum_{k=1}^{q} \sum_{l=1}^{q} \theta_{k}\left(z\left(i h_{0}+j h\right)\right) \\
& \times \theta_{l}\left(z\left(i h_{0}+j h\right)\right)\left(G_{k}+H_{k} K_{k l}\right) x_{d}\left(i h_{0}+j h\right)(11)
\end{aligned}
$$

Replacing $j$ in (11) to $j-1$ leads

$$
\begin{aligned}
& x_{d}\left(i h_{0}+j h\right)=\sum_{k=1}^{q} \sum_{l=1}^{q} \theta_{k}\left(z\left(i h_{0}+j h-h\right)\right) \\
& \times \theta_{l}\left(z\left(i h_{0}+j h-h\right)\right)\left(G_{k}+H_{k} K_{k l}\right) x_{d}\left(i h_{0}+j h-h\right)
\end{aligned}
$$

We compute

$$
\begin{aligned}
x_{d}\left(i h_{0}+h\right)= & \sum_{k=1}^{\mathrm{q}} \sum_{l=1}^{\mathrm{q}} \theta_{k}\left(z\left(i h_{0}\right)\right) \theta_{l}\left(z\left(i h_{0}\right)\right) \\
& \times\left(G_{k}+H_{k} K_{k l}\right) x_{d}\left(i h_{0}\right) \\
x_{d}\left(i h_{0}+2 h\right)= & \sum_{k=1}^{q} \sum_{l=1}^{q} \theta_{k}\left(z\left(i h_{0}+h\right)\right) \\
& \times \theta_{l}\left(z\left(i h_{0}+h\right)\right)\left(G_{k}+H_{k} K_{k l}\right) \\
& \times x_{d}\left(i h_{0}+h\right)
\end{aligned}
$$

$$
\begin{aligned}
= & \sum_{k_{0}=1}^{q} \sum_{l_{0}=1}^{q} \sum_{k_{1}=1}^{q} \sum_{l_{1}=1}^{q} \theta_{k_{0}}\left(z\left(i h_{0}+h\right)\right) \\
& \times \theta_{l_{0}}\left(z\left(i h_{0}+h\right)\right) \theta_{k_{1}}\left(z\left(i h_{0}\right)\right) \\
& \times \theta_{l_{1}}\left(z\left(i h_{0}\right)\right)\left(G_{k_{0}}+H_{k_{0}} K_{k_{0} l_{0}}\right) \\
& \times\left(G_{k_{1}}+H_{k_{1}} K_{k_{1} l_{1}}\right) x_{d}\left(i h_{0}\right)
\end{aligned}
$$

for $\left(k_{0}, j_{0}, k_{1}, j_{1}\right) \in \underbrace{I_{q} \times \cdots \times I_{q}}_{4}$. Proceeding forward, we can readily obtain (10) for $j>0$.

Substituting (10) to $x_{d}\left(i h_{0}+j h\right)$ in (9) controlled by (8), we can obtain the followingdiscretized version of the closed-loop digital fuzzy system with (7) and (8):

$$
\begin{aligned}
x_{d}\left(i h_{0}+j h+h\right)= & \prod_{v=0}^{j}\left(\sum_{k=1}^{q} \sum_{l=1}^{q} \theta_{k}\left(z\left(i h_{0}+j h-v h\right)\right)\right. \\
= & \left.\theta_{l}\left(z\left(i h_{0}+j h-v h\right)\right)\left(G_{k}+H_{k} K_{k l}\right)\right) \\
& \times x_{d}\left(i h_{0}\right)
\end{aligned}
$$

for $i \in Z_{0}$ and $j \in Z_{[0, N-1]}$.

Corollary 2. In the the analogously controlled fuzzy system (6),

- the approximate discrete-time model can be also obtained as

$$
\begin{aligned}
x_{c}\left(i h_{0}+j h+h\right)= & \sum_{k=1}^{q} \sum_{l=1}^{q} \theta_{k}\left(z\left(i h_{0}+j h\right)\right) \\
& \times \theta_{l}\left(z\left(i h_{0}+j h\right)\right) \Xi_{k l} x_{c}\left(i h_{0}+j h\right)
\end{aligned}
$$

where $\Xi_{k l}=\mathrm{e}^{\left(A_{k}+B_{k} \bar{K}_{l}\right) h}$.

- the solution to (13) for $x_{c}\left(i h_{0}+j h\right)$ with the arbitrary initial state $x_{c}\left(i h_{0}\right)$ is given by

$$
\begin{aligned}
x_{c}\left(i h_{0}+j h\right)= & \prod_{v=1}^{j}\left(\sum_{k=1}^{q} \sum_{l=1}^{q} \theta_{k}\left(z\left(i h_{0}+j h-v h\right)\right)\right. \\
& \left.\times \theta_{l}\left(z\left(i h_{0}+j h-v h\right)\right) \Xi_{k l}\right) x_{c}\left(i h_{0}\right)
\end{aligned}
$$

for $i \in Z_{0}$ and $j \in Z_{[1, N-1]}$.

Therefore, from (13) and (14), we directly obtain the following discrete-time representation of (6):

$$
\begin{aligned}
x_{c}\left(i h_{0}+j h+h\right)= & \prod_{v=0}^{j}\left(\sum_{k=1}^{q} \sum_{l=1}^{q} \theta_{k}\left(z\left(i h_{0}+j h-v h\right)\right)\right. \\
& \left.\times \theta_{l}\left(z\left(i h_{0}+j h-v h\right)\right) \Xi_{k l}\right) x_{c}\left(i h_{0}\right)
\end{aligned}
$$


for $i \in Z_{0}$ and $j \in Z_{[1, N-1]}$.

\subsection{Design of the periodic controller using IDR method}

The IDR problem for the system (8) is the problem to design a periodic control law (9) such that i) the origin $x=0$ is a globally asymptotically stable equilibrium point of the closed-loop system

$$
\begin{aligned}
x_{d}(t)= & \sum_{k=1}^{q} \sum_{l=1}^{q} \theta_{k}(z(t)) \theta_{l}\left(z\left(i h_{0}+j h\right)\right) \\
& \times\left(A_{k} x_{d}(t)+B_{k} K_{k l} x_{d}\left(i h_{0}+j h\right)\right),
\end{aligned}
$$

and ii) by comparing (12) and (15), to realize $x_{c}\left(i h_{0}+h\right)=x_{d}\left(i h_{0}+h\right)$ under the assumption that $x_{c}\left(i h_{0}\right)=x_{d}\left(i h_{0}\right), K_{k l}$ was numerically synthesized for $\left\|\Xi_{k l}-G_{k}-H_{k} K_{k l}\right\|$ to be a minimizer in the induced 2 -norm sense.

Theorem 1. If there exist $Q=Q^{T} \succ 0$ and constant matrices $F_{k}$ such that the following generalized eigenvalue problem (GEVP) has solutions:

${\text { Minimize } Q, F_{k}} \gamma$ subject to

$$
\begin{aligned}
& {\left[\begin{array}{cc}
-\gamma Q & (\bullet)^{T} \\
\Xi_{k l} Q-G_{k} Q-H_{k} F_{k l} & -\gamma I
\end{array}\right] \prec 0, \quad k, l \in I_{q}} \\
& {\left[\begin{array}{cc}
-Q & (\bullet)^{T} \\
G_{k} Q+H_{k} F_{k k} & -Q
\end{array}\right] \prec 0, \quad k, l \in I_{q}} \\
& {\left[\begin{array}{cc}
-Q & (\bullet)^{T} \\
\frac{G_{k} Q+H_{k} F_{k J}+G_{l} Q+H_{l} F_{l k}}{2} & -Q
\end{array}\right] \prec 0, \quad k, l \in I_{q}}
\end{aligned}
$$

then the state $x_{d}\left(i h_{0}+j h\right)$ of the discrete-time representation (12) closely matches the discrete-time representation (15), and (12) is globally asymptotically stable in the sense of Lyapunov, where $(\bullet)^{T}$ denotes the transposed element in symmetric positions.

\section{Computer Simulations: DUFFING-LIKE CHAOTIC OSCILLATOR}

In this section, we use the results in Section 3 to digitally control the continuous-time $\mathrm{T}-\mathrm{S}$ fuzzy system, which is the fuzzy model of the duffing-like chaotic oscillator [6]. The IF-Then rule of the duffing-like chaotic oscillator is given by

$$
R_{1}: \text { IF } x_{1}(t) \text { is about } \Gamma_{11}
$$

$$
\text { THEN } \frac{d}{d t}\left[\begin{array}{l}
x_{1}(t) \\
x_{2}(t) \\
x_{3}(t) \\
x_{4}(t)
\end{array}\right]=A_{1}\left[\begin{array}{l}
x_{1}(t) \\
x_{2}(t) \\
x_{3}(t) \\
x_{4}(t)
\end{array}\right]
$$

$R_{2}:$ IF $x_{1}(t)$ is about $\Gamma_{21}$

$$
\text { THEN } \frac{d}{d t}\left[\begin{array}{l}
x_{1}(t) \\
x_{2}(t) \\
x_{3}(t) \\
x_{4}(t)
\end{array}\right]=A_{2}\left[\begin{array}{l}
x_{1}(t) \\
x_{2}(t) \\
x_{3}(t) \\
x_{4}(t)
\end{array}\right]
$$

where

$$
A_{1}=\left[\begin{array}{cccc}
0 & 1 & 0 & 0 \\
1.1 & -0.3 & 2.1 & 0 \\
0 & 0 & 0 & 0.5 \pi \\
0 & 0 & -0.5 \pi & 0
\end{array}\right], \quad A_{2}=\left[\begin{array}{cccc}
0 & 1 & 0 & 0 \\
-1.4 & -0.3 & 2.1 & 0 \\
0 & 0 & 0 & 0.5 \pi \\
0 & 0 & -0.5 \pi & 0
\end{array}\right]
$$

and the membership functions are

$$
\left\{\begin{array}{l}
\Gamma_{1}^{1}\left(x_{1}(t)\right)=1-\left|x_{1}(t)\right| / 2.1 \\
\Gamma_{1}^{2}\left(x_{1}(t)\right)=\left|x_{1}(t)\right| / 2.1
\end{array}\right.
$$

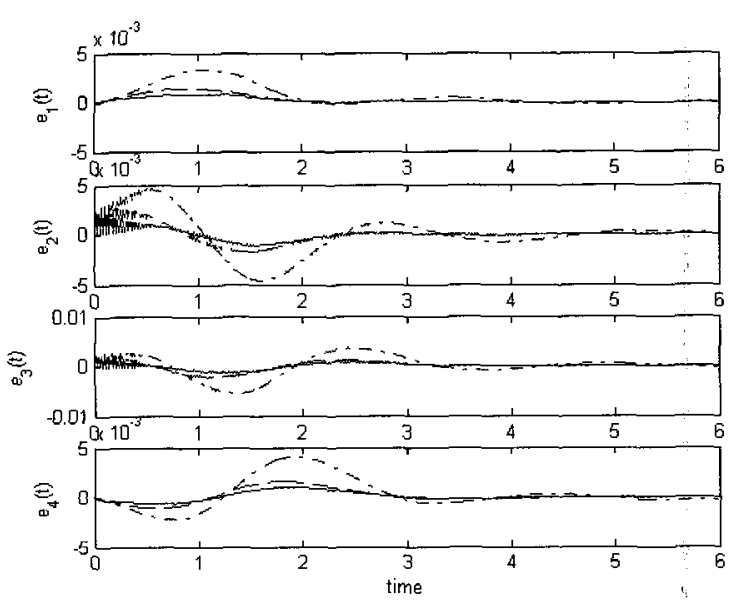

Figure 1. Simulation results for $0.03(\mathrm{~s})$, showing the error in the state trajectories between the continuoustime control and the sampled-data control schemes using the proposed method (solid), [1] (dashed), and [6] (dash-dotted)

First, we simulate the continuous-time $T-S$ fuzzy system. The input matrices are arbitrary chosen as

$$
B_{1}=B_{2}=\left[\begin{array}{l}
0 \\
1 \\
1 \\
0
\end{array}\right]
$$

where preserve the controllability of the system. To match the trajectories of the closed-loop analog fuzzy system with the following analog control gain

$$
\begin{aligned}
& K_{c 1}=[-5.8725-2.4098-1.4306-0.0692] \\
& K_{c 2}=[-5.2303-3.3683-0.8664-0.4055]
\end{aligned}
$$

we employ the Theorem 1 to design the single-rate 
sampled-data controller for sampling periods $0.03(\mathrm{~s})$, $1(\mathrm{~s})$, and $1.5(\mathrm{~s})$. We can obtain the following feasible sampled-data control gain:

For $0.03(\mathrm{~s})$

$$
\begin{aligned}
& K_{11}=[-5.5788-2.3475-1.4217-0.0987] \\
& K_{21}=[-5.4904-2.3466-1.4217-0.0987] \\
& K_{12}=[-4.9590-3.2197-0.9057-0.4013] \\
& K_{22}=[-4.8370-3.2185-0.9056-0.4013]
\end{aligned}
$$

For 1(s)

$$
\begin{array}{r}
K_{11}=[-1.0022-0.8026-0.8066-0.7008] \\
K_{21}=[0.1847-0.4201-0.6685-0.7689] \\
K_{12}=[-0.9543-0.7847-0.7948-0.6585] \\
K_{22}=[0.2439-0.4156-0.6691-0.7024]
\end{array}
$$

For $1.5(\mathrm{~s})$

$$
\begin{gathered}
K_{11}=[-0.7195-0.6043-0.4612-0.7584] \\
K_{21}=[0.1610-0.1976-0.1470-0.8449] \\
K_{12}=[-0.6920-0.5852-0.5322-0.7158] \\
K_{22}=[0.3790-0.1187-0.2286-0.7840]
\end{gathered}
$$

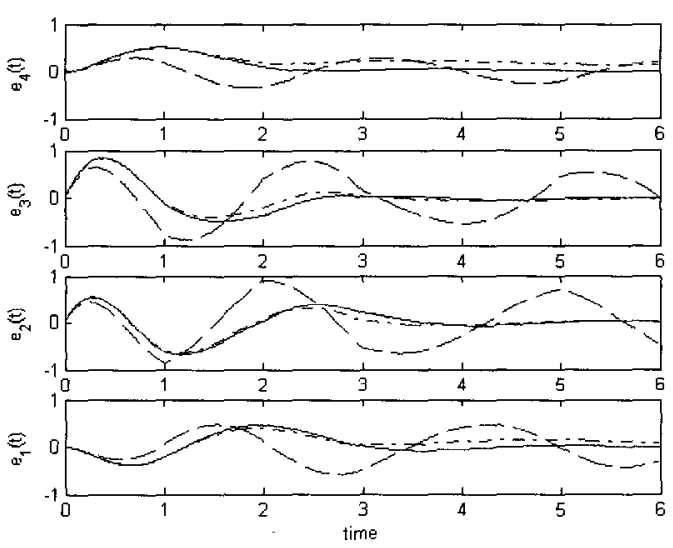

Figure 2. Simulation results for $1(\mathrm{~s})$, showing the error in the state trajectories between the continuous-time control and the sampled-data control schemes using the proposed method (solid), [1] (dashed), and [6] (dash-dotted)

Compared with previous results[1],[6], our proposed digital redesign method is the smallest error between the closed-loop analog and sampled systems as shown in Figures 1, 2, and 3. For quantitative comparison of the performance of the proposed intelligent digital redesign technique with the other method, the performance measure is defined as

$$
J=\sum_{i=1}^{n}\left(\int_{0}^{6}\left|x_{c_{i}}(t)-x_{d_{i}}(t)\right| d t\right)
$$

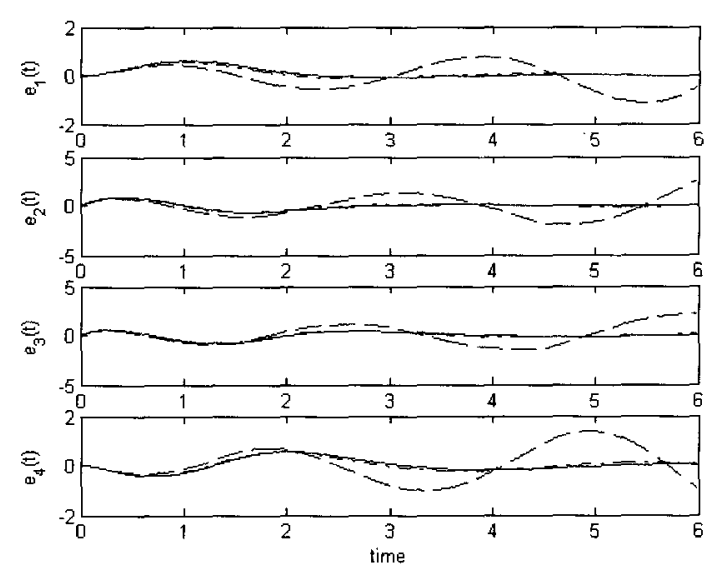

Figure 3. Simulation results for $1.5(\mathrm{~s})$, showing the error in the state trajectories between the continuous-time control and the sampled-data control schemes using the proposed method (solid), [1] (dashed), and [6] (dash-dotted)

The performance comparison of them is shown in Table 1, which indicate that the proposed method outperforms that of Joo[1] and Lee[6].

Table 1. Perfomance Comparison

\begin{tabular}{|c|l|l|l|}
\hline \multirow{2}{*}{ Method } & \multicolumn{3}{|c|}{ Sampling period } \\
\cline { 2 - 4 } & $0.03(\mathrm{~s})$ & $1(\mathrm{~s})$ & $1.5(\mathrm{~s})$ \\
\hline Proposed Method & 0.0068 & 3.8692 & 4.9798 \\
\hline Joo [1] & 0.0109 & 8.0441 & 16.4867 \\
\hline Lee [6] & 0.0289 & 4.4644 & 5.1406 \\
\hline
\end{tabular}

\section{Conclusions}

This paper proposed the periodic control design using the LMI approach for the fuzzy system. Some sufficient conditions were derived for stabilization and state matching of the discretized model by the fast discretization. The proposed periodic control scheme can improve the state-matching performance in the long sampling limit.

\section{References}

[1] Y. H. Joo, L. S. Shieh, and G. Chen, "Hybrid state space fuzzy model-based controller with dual-rate sampling for digital control of chaotic systems," IEEE Trans. Fuzzy Syst., vol. 7, no. 4, pp. 394-408, 1999.

[2] W. Chang, J. B. Park, Y. H. Joo, and G. Chen, "Design of sampled-data fuzzy-model-based control systems by using intelligent digital redesign," IEEE Trans. Circ. Syst. I, vol. 49, no. 4, pp. 509-517, 2002.

[3] W. Chang, J. B. Park, and Y. H. Joo, "GA-based 
intelligent digital redesign of fuzzy-model-based controllers," IEEE Trans. Fuzzy Syst., vol. 11, no. 1, pp. 35-44, 2003.

[4] W. Chang, J. B. Park, H. J. Lee, and Y. H. Joo, "LMI approach to digital redesign of linear time-invarint systems," IEE Proc., Control Theory Appl., vol. 149, no. 4, pp. 297-302, 2002.

[5] H. J. Lee, J. B. Park, and Y. H. Joo, "An efficient observer-based sampled-data control: Digital redesign approach," IEEE Trans. Circuits Syst. I, vol. 50, no. 12, pp. 1595-1601, 2003.

[6] H. J. Lee, H. Kim, Y. H. Joo, W. Chang, and J. B. Park, "A new intelligent digital redesign for $\mathrm{T}-\mathrm{S}$ fuzzy systems: global approach," IEEE Trans. Fuzzy Syst., vol. 12, no. 2, pp. 274-284, 2004.

[7] T. Chen and B. Francis, "Optimal Sampled-Data Control Systems," Springer, 1995.

[8] B. A. Francis and T. T. Georgiou, "Stability theory for linear time-invariant plants with periodic digital controllers," IEEE Trans. Automat. Contr., vol. 33, no. 9, pp. 820-832, 1988.

[9] L. S. Shieh, W. M. Wang, J. Bain, and J. W. Sunkel, "Design of lifted dual-rate digital controllers for X-38 vehicle," Jounal of Guidance, Contr. Dynamics, vol. 23, pp. 629-339, Jul., 2000.

[10] H. O. Wang, K. Tananka, and M. F. Griffin, "An approach to fuzzy control of nonlinear systems: Stability and design issues," IEEE Trans. Fuzzy Syst., vol. 4, no. 1, pp. 14-23, 1996.

[11] K. Tananka, T. Kosaki, and H. O. Wang, "Backing control problem of a mobile robot with multiple trailers: fuzzy modeling and LMI-based design," IEEE Trans. Syst. Man, Cybern. C., vol. 28, no. 3, pp. 329-337, 1998.

[12] H. Fujimoto, Y. Hori, and A. Kawamura, "Perfect tracking control based on multirate feedforward control with generalized sampling periods," IEEE Trans. Ind. Electron., vol. 48, no. 3, pp. 636-644, 2001.

[13] T. Chen and L. Qiu, "Ho design of general multirate sampled-data control systems," Automatica, vol. 30, pp. 1139-1152, 1994.

[14] L. Qiu and T. Chen, "H2 -optimal design of multirate sampled-data systems," IEEE Trans. Automat. Contr., vol. 39, pp. 2506-2511, 1994.

[15] P. Colaneri and G. De Nicolao, "Multirate LQG control of continuous - time stochastic systems," Automatica, vol. 31, pp. 591-596, 1995.

[16] P. G.Voulgaris and B. Bamieh, "Optimal $\mathrm{H} \infty$ and $\mathrm{H} \in$ control of hybrid multirate systems," Syst. Contr. Lett., vol. 20, pp. 249-261, 1993.
[17] P. G. Voulgaris, M. A. Dahleh, and L. S. Valavani, "Hळ and $\mathrm{H} \in$ optimal controllers for periodic and multirate systems," Automatica, vol. 30, pp. 251-263, 1994.

[18] H. Bo and A. N. Michel, "Some qualitative properties of multirate digital control systems," IEEE Trans. Automat. Contr., vol. 44, pp. $765-770$, 1999.

[19] G. J. Pappas and S. Simic , "Consistent abstractions of affine control systems," IEEE Trans. Automat. Contr., vol. 47, pp. 745 - 756, 2002.

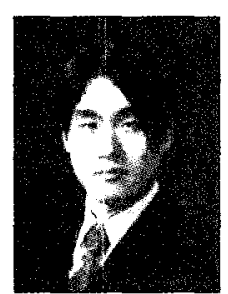

김도완(Do Wan Kim)

제 14권 2호(2004년 4월호) 참조

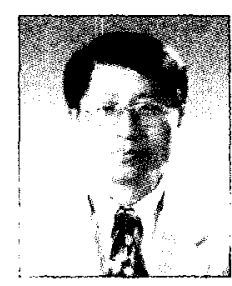

주영훈(Young Hoon Joo)

제 14권 2호(2004년 4월호) 참조

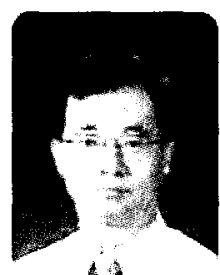

박진배 (Jin Bae Park)

제 14권 2호(2004년 4월호) 참조 